\title{
Wunderlich syndrome as the first manifestation of an extraskeletal Ewing sarcoma
}

\author{
Jong Wook Kim, MD; Ji Yun Chae, MD; Cheol Yong Yoon, MD; Mi Mi Oh, MD; Hong Seok Park, MD; \\ Du Geon Moon, MD
}

Department of Urology, Korea University Guro Hospital, Seoul, Korea

Cite as: Can Urol Assoc J 2015;(9-10):E648-50. http://dx.doi.org/10.5489/cuaj.2411

Published online September 9, 2015.

\section{Abstract}

We recently encountered an extremely rare case of spontaneous perirenal hemorrhage in a 34-year-old man. He initially had undergone radical nephrectomy owing to suspicion of renal cell carcinoma. The final diagnosis was extraskeletal Ewing sarcoma.

\section{Introduction}

Ewing sarcoma (ES) is a poorly differentiated, aggressive malignancy that most frequently develops in bone, soft tissues, and the chest wall. Although the kidney has been increasingly recognized as an unexpected site for extraskeletal ES, ${ }^{1}$ primary renal ES is still uncommon, furthermore, spontaneous rupture of primary renal ES is rarely reported. We present an extremely rare case of spontaneous perirenal hemorrhage because of renal ES.

\section{Case report}

A 34-year-old man presented to the emergency department with left flank pain for 1 day. He had no history of specific disease or trauma. Physical examination indicated moderate tenderness across the whole abdomen, which was more severe on the left side. No rebound tenderness was noted in the abdomen. However, severe costovertebral angle tenderness was present on the left side.

He had a blood pressure of 140/90 $\mathrm{mmHg}$, pulse rate of 95 beats/min, respiratory rate of 20 breaths/min, and body temperature of $37.7^{\circ} \mathrm{C}$. A complete blood count was performed, the results of which revealed a hemoglobin of $14.3 \mathrm{~g} /$ $\mathrm{dL}$, a platelet count of $176000 / \mu \mathrm{L}$, a blood urea nitrogen level of $18.5 \mathrm{mg} / \mathrm{dL}$, and a creatinine level of $0.92 \mathrm{mg} / \mathrm{dL}$.

An urgent contrast-enhanced computed tomography (CT) scan was performed (Fig. 1). We visualized a suspicious $6.8-\mathrm{cm}$ mass originating from the upper pole of the left kidney, with internal necrosis and hemorrhage in the left perirenal space. The first impression of the radiologist was spontaneous rupture of renal cell carcinoma (RCC), and the second was fat depletion angiomyolipoma. On the third day of hospitalization, we performed a radical nephrectomy due to potential of malignancy.

Macroscopic examination revealed a ruptured tumour mass with circumjacent hemorrhage, and the ruptured surface showed hemorrhagic and adipose tissue. Microscopically, the tumours were in the renal pelvis and ureter, infiltrating to the renal parenchyma (Fig. 2a). They consisted of uniform, small round cells with round nuclei containing fine chromatin, scanty clear, or eosinophilic cytoplasm, and indistinct cytoplasmic membranes (Fig. 2b). In the immunohistochemical analysis, the cells exhibited diffuse membranous positive staining with CD99 and vimentin, while cytokeratin was negative (Fig. 2c). The EWS-FLI1 fusion gene consistent with $\mathrm{t}(11 ; 22)$ demonstrated by fluorescence in situ hybridization study (Fig. 2d). The pathologic diagnosis was high-grade extraskeletal ES.

In addition, we performed a chest CT scan, whole body positron emission tomography (PET)-CT scan, and whole body bone scan that revealed no other pathologic site (primary origin). The final diagnosis was spontaneous perirenal hemorrhage of primary renal ES. He was discharged 10 days after nephrectomy, and received adjuvant chemotherapy (3 cycles of vincristine, adriamycin, cyclophosphamide, ifosfamide and etoposide). Seven months after the procedure, the patient remained stable with no evidence of tumour recurrence.

\section{Discussion}

Wunderlich syndrome is a spontaneous, non-traumatic, subcapsular or perirenal hemorrhage. In 1856 Wunderlich first described the condition of spontaneous renal bleeding with dissection of blood into the subcapsular and/or perinephric spaces. A meta-analysis of Wunderlich syndrome treatments showed that $83 \%$ of patients presented with acute onset of flank pain, 19\% had hematuria, and 


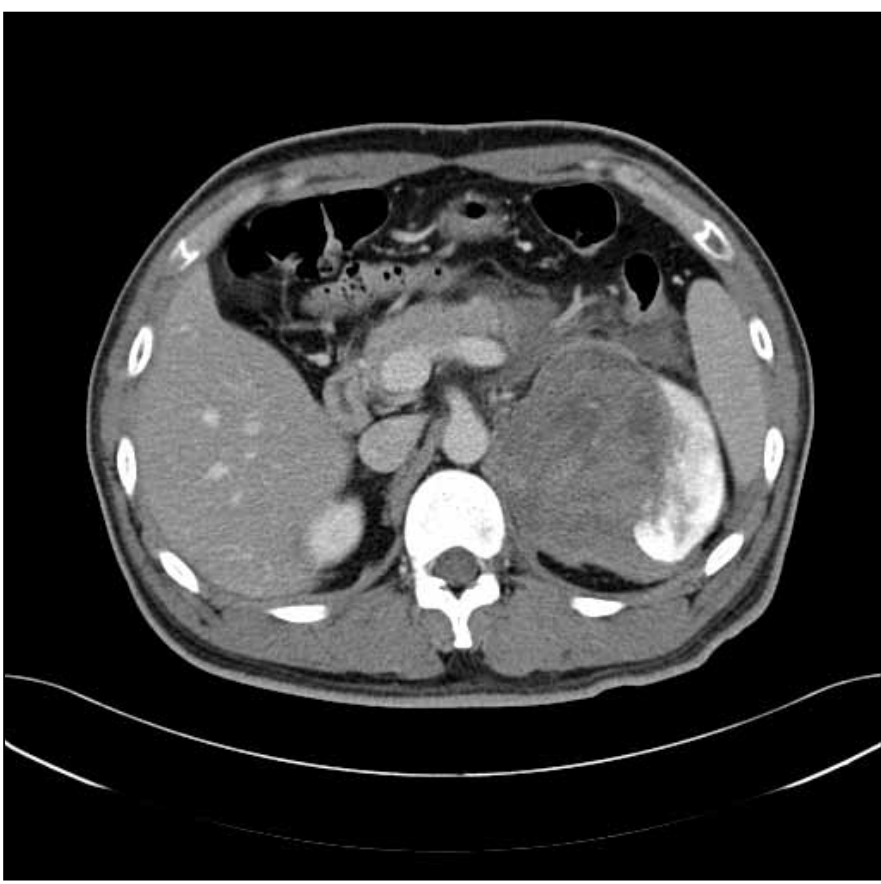

Fig. 1. Computed tomography scan showing left perinephric hemorrhage with extravasation and a roughly $6.8-\mathrm{cm}$-sized mass with internal necrosis and hemorrhage in the left perirenal space.

$11 \%$ had symptoms and signs of hypovolemic shock. Benign and malignant neoplasms accounted for most cases $(61.5 \%)$, with angiomyolipoma being predominant $(29.1 \%)$, followed closely by RCC (26.1\%). Many urologists believe that because of the high likelihood of underlying malignancy, exploratory surgery or nephrectomy is necessary in patients with Wunderlich syndrome. ${ }^{2}$

Cases of Wunderlich syndrome resulting from ES are extremely rare. ES and peripheral primitive neuroectodermal

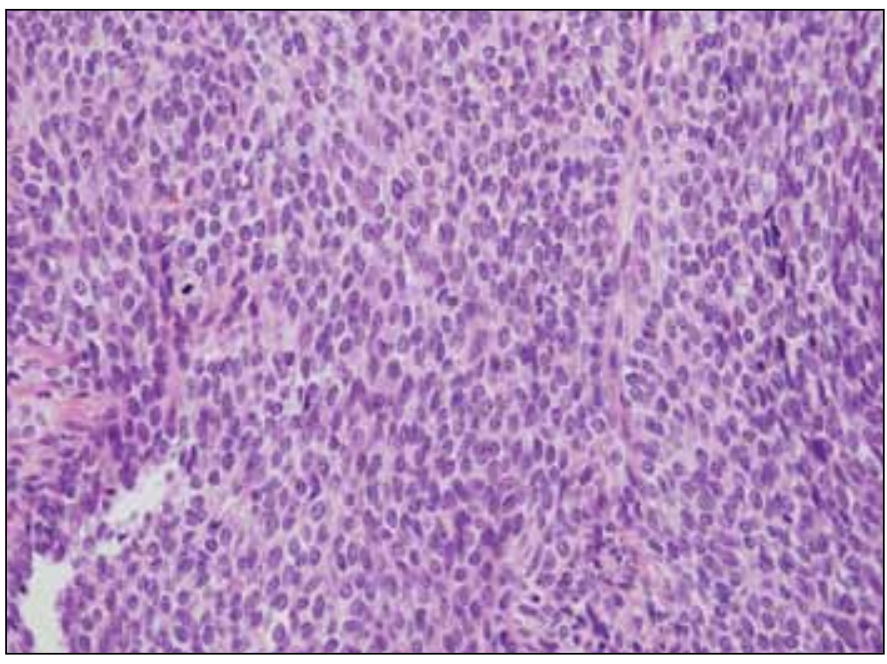

Fig. 2b. Histopathological examination of primary renal Ewing sarcoma. The tumours included uniform, small round cells with round nuclei containing fine chromatin, scanty clear, or eosinophilic cytoplasm, and indistinct cytoplasmic membranes.

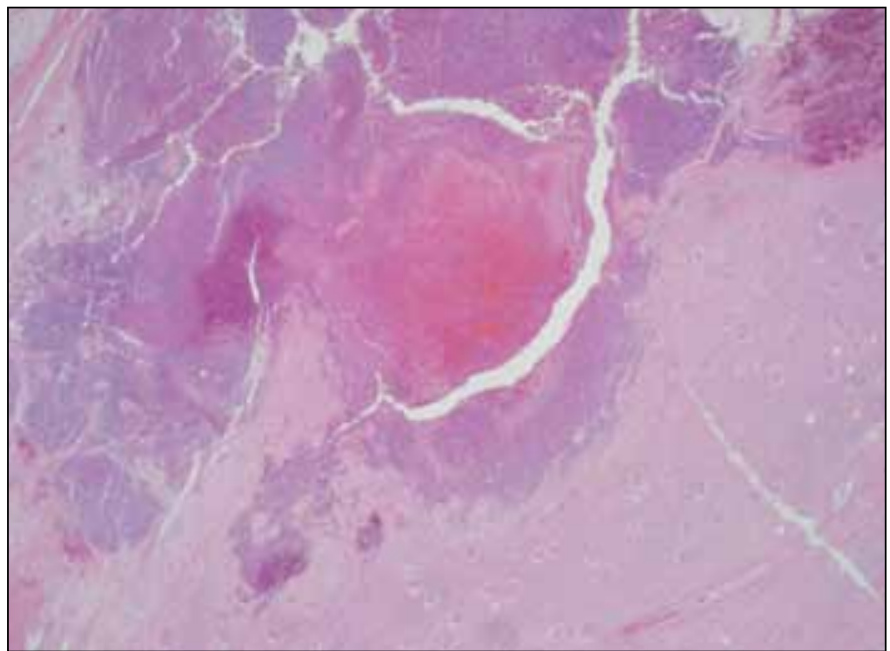

Fig. 2a. Histopathological examination of primary renal Ewing sarcoma. The tumours were in the renal pelvis and ureter, infiltrating to the renal parenchyma.

tumour are now regarded as two morphological ends of a spectrum of neoplasms. Primary extraskeletal ES are uncommon and have been reported in various sites, usually in the chest wall, lower extremities, and the paravertebral region. ${ }^{3}$ However, the kidney has been increasingly recognized as an unexpected site for ES. Frequently, after radiographic identification of a renal mass in adults, the presumptive diagnosis is RCC, and radical nephrectomy is undertaken. It can be difficult to distinguish a case of ES from RCC. ${ }^{1}$ However, Hakky and colleagues discovered that renal ES commonly presents with symptoms, while RCC is an incidental finding on imaging. ${ }^{4}$

In our case, the patient initially underwent radical nephrectomy because of the high likelihood of RCC. The

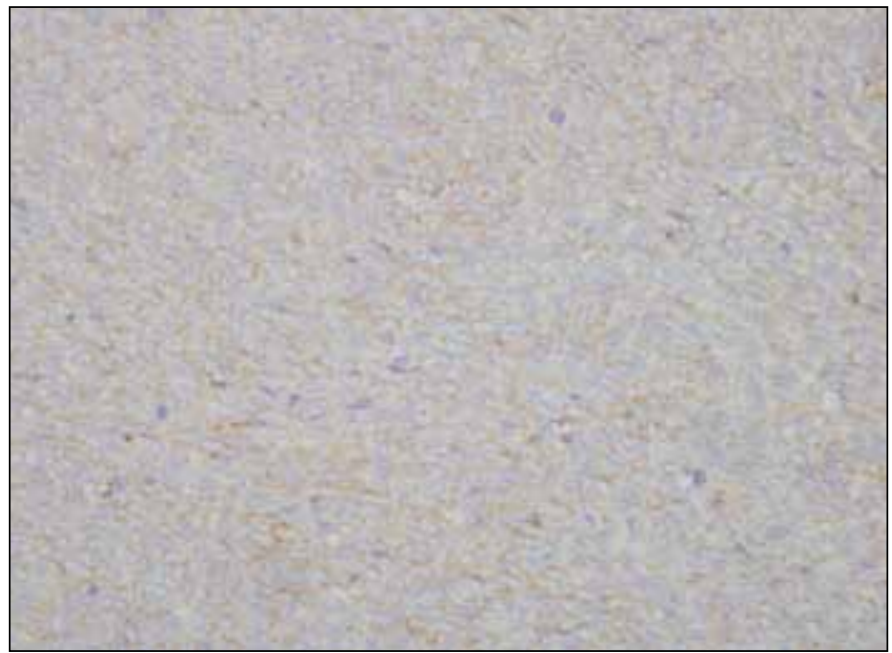

Fig. 2c. Histopathological examination of primary renal Ewing sarcoma. In the immunohistochemical analysis, the cells exhibited diffuse membranous positive staining with CD99 and vimentin, while cytokeratin was negative. 
Kim et al.

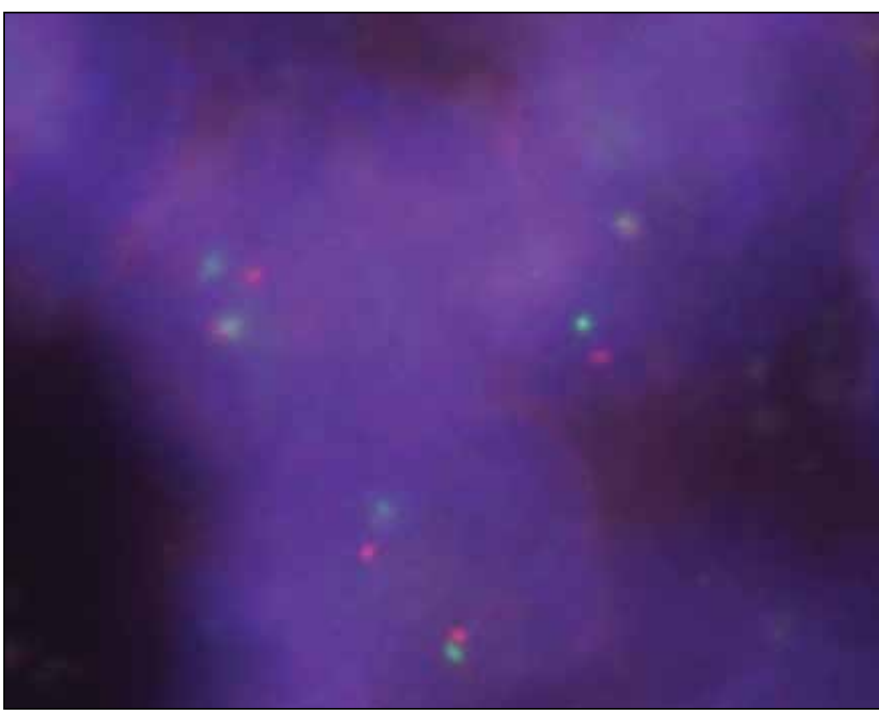

Fig. 2d. Histopathological examination of primary renal Ewing sarcoma. The EWS-FLI1 fusion gene consistent with $\mathrm{t}(11 ; 22)$ demonstrated by fluorescence in situ hybridization study.

final diagnosis of ES was confirmed through immunohistochemical analysis for cytokeratin, vimentin, and CD99. Current evidence-based management of ES emphasizes the importance of a multidisciplinary approach to treatment, with both local control (surgery, radiotherapy, or these modalities in combination) and systemic multi-agent chemotherapy, as essential components of the current standard of care. ${ }^{1}$ However, the survival rate in previous reports remains poor. ${ }^{5}$

\section{Conclusion}

As only one case of Wunderlich syndrome with primary renal ES has been previously reported, this case report is a valuable addition to the limited literature in this area. We should consider this diagnosis especially in selected young patients who present with sudden flank pain.

Competing interests: The authors all declare no competing financial or personal interests.

This paper has been peer-reviewed.

\section{References}

1. Rowe RG, Thomas DG, Schuetze SM, et al. Ewing sarcoma of the kidney: Case series and literature review of an often overlooked entity in the diagnosis of primary renal tumors. Urology 2013;81:347-53. http:// dx.doi.org/10.1016/i.urology.2012.10.016

2. Zhang JQ, Fielding JR, Zou KH. Etiology of spontaneous perirenal hemorrhage: A meta-analysis. J Urol 2002;167:1593-6. http://dx.doi.org/10.1016/S0022-5347(05)65160-9

3. Che SM, Cao PL, Chen HW, et al. Primary Ewing's sarcoma of vulva: A case report and a review of the literature. J Obstet Gynaecol Res 2013;39:746-9. http://dx.doi.org/10.1111/i.1447-0756.2012.02019.x

4. Hakky TS, Gonzalvo AA, Lockhart JL, et al. Primary Ewing sarcoma of the kidney: A symptomatic presentation and review of the literature. Ther Adv Urol 2013;5:153-9. http://dx.doi. org/10.1177/1756287212471095

5. Moustafellos $P$, Gourgiotis $S$, Athanasopoulos $G$, et al. A spontaneously ruptured primitive neuroectodermal tumor/extraosseous Ewing's sarcoma of the kidney with renal vein tumor thrombus. Int Urol Nephrol 2007;39:393-5. http://dx.doi.org/10.1007/s1 1255-006-9073-x

Correspondence: Dr. Du Geon Moon, Department of Urology, Korea University Guro Hospital, Korea University Institute of Regenerative Medicine, \#1 48 Gurodong-ro, Guro-gu, Seoul 152-703, Korea; dgmoon@korea.ac.kr 